Chirurg 2021 -92:961

https://doi.org/10.1007/s00104-021-01507-5

Angenommen: 25. August 2021

Online publiziert: 9 . September 2021

(c) Springer Medizin Verlag $\mathrm{GmbH}$, ein Teil von Springer Nature 2021

\section{Resektionsausmaß bei Kolonkarzinomen im Bereich der linken Kolonflexur}

\author{
Ergebnisse einer Netzwerkmetaanalyse
}

\section{T. Germer · J. Reibetanz}

Klinik für Allgemein-, Viszeral-, Transplantations-, Gefäß- und Kinderchirurgie, Universitätsklinik Würzburg, Würzburg, Deutschland

\section{Originalpublikation}

Wang $X$ et al (2021) Subtotal colectomy, extended right hemicolectomy, left hemicolectomy, or splenic flexure colectomy for splenic flexure tumors: a network meta-analysis. Int J Colorectal Dis 36(2):311-322. https://doi.org/ 10.1007/s00384-020-03763-z.

Einleitung und Zielsetzung. Aus chirurgischer Sicht ist die Lokalisation der Karzinome der linken Kolonflexurv.a. deswegen interessant, da sie sich im Bereich der "Wasserscheide" des Stromgebietes der Arteria mesenterica superior und der Arteria mesenterica inferior befinden. Entsprechend den onkologischen Prinzipien der kurativen Primärtumorresektion konkurrieren daher unterschiedliche Resektionsausmaße für die Karzinome der linken Kolonflexur: Die subtotale Kolektomie (STK), die erweiterte Hemikolektomie rechts (EHKR), die Hemikolektomie links (HKL) und die Resektion der linken Kolonflexur (FRL). Ziel der aktuellen Netzwerkmetaanalyse war es, die vorhandene Literatur bezüglich dieser Resektionsausmaße hinsichtlich Kurzzeitergebnissen und onkologischem Outcome auszuwerten. Hierzu wurden aus den bekannten medizinischen Datenbanken alle relevanten Originalpublikationen bis August 2020 extrahiert, die mindestens 2 der oben genannten Resektionsausmaße bei Patienten mit Kolonkarzinom der linken Flexur miteinander verglichen.

Ergebnisse. Insgesamt konnten 10 Studien mit $N=2734$ Patienten mit Karzinomen der linken Kolonflexur identifiziert werden: $N=109$ Patienten im STK-Arm, $N=596 \mathrm{~Pa}-$ tienten im EHKR-Arm, $N=928$ Patienten im HKL-Arm und $N=1101$ Patienten im FRLArm. Der Anteil männlicher Patienten betrug 57,4\%, das mittlere Alter schwankte zwischen 63 und 74 Jahren und der Anteil laparoskopisch durchgeführter Operationen zwischen 11,2 und 85,3\%. Das Risiko einer Anastomoseninsuffizienz war für die STC am geringsten und für die FRL am höchsten, jedoch nicht signifikant unterschiedlich. Gleiches galt für den Endpunkt „perioperative Mortalität“. Die Lokalrezidivrate unterschied sich zwischen den einzelnen Techniken nicht signifikant, mit allerdings dem höchsten Risiko eines Lokalrezidivs für die FRL und dem niedrigsten Risiko für die STK. Die Rate an Metastasierung war für die einzelnen Operationsverfahren vergleichbar. Daten zum krankheitsfreien Überleben, rezidivfreien Überleben und progressionsfreien Überleben wurden nur von 4 Studien berichtet. Somit war eine Metaanalyse hinsichtlich dieser Endpunkte nicht möglich. Die Einzelauswertung dieser Studien zeigte aber keinen signifikanten Unterschied zwischen FRL, HKL und EHKR in Bezug auf das krankheitsfreie Überleben. Das Gesamtüberleben (OS) wurde von 7 Studien mit $N=1055$ Patienten analysiert. Hierbei resultierte die STK im besten OS, gefolgt von HKL, FRL und EHKR.

Fazit des Reviewers. Die vorliegende Netzwerkmetaanalyse zeigt auf hohem Evidenzniveau, dass die 4 möglichen Resektionsverfahren für Karzinome der linken Kolonflexur in einer vergleichbaren Lymphknotenausbeute und in vergleich- baren Überlebensdaten resultieren. Obwohl die EHKR mit einem höheren Risiko für einen postoperativen lleus einherging, zeigte sich für dieses Resektionsausmaß eine höhere Rate an primärer Anastomose und ein (nicht-signifikanter) Trend zu einer niedrigeren Anastomoseninsuffizienzrate, verglichen mit den übrigen, limitierteren Resektionsverfahren. Ein eindeutig zu bevorzugendes, da überlegenes Resektionsverfahren lässt sich nicht definieren.

\section{Korrespondenzadresse}

\section{Dr. J. Reibetanz}

Klinik für Allgemein-, Viszeral-, Transplantations-, Gefäß- und Kinderchirurgie, Universitätsklinik Würzburg 97080 Würzburg, Deutschland Reibetanz_j@ukw.de

Interessenkonflikt. C.T. Germer und J. Reibetanz geben an, dass kein Interessenkonflikt besteht.

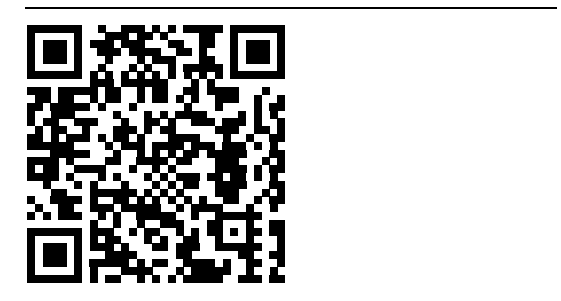

QR-Code scannen \& Beitrag online lesen 